\title{
Analyse et renforcement du programme de sécurité des vaccins au Manitoba
}

\author{
Montalban $\mathrm{JM}^{1^{*}}$, Ogbuneke $\mathrm{C}^{2}$, Hilderman $\mathrm{T}^{1,3}$
}

${ }^{1}$ Direction générale de la santé publique, Santé Manitoba et Vie saine, Aînés et Consommation, Winnipeg (Manitoba)

${ }^{2}$ Santé publique et médecine préventive, École de médecine du Nord de I'Ontario, Sudbury (Ontario)

${ }^{3}$ Sciences de la santé communautaire, Université du Manitoba, Faculté de médecine, Winnipeg (Manitoba)

*Correspondance: joselito.montalban@gmail.com

\section{Résumé}

Contexte : L'apparition d'un nouveau virus grippal A en 2009 et l'introduction rapide de nouveaux vaccins pandémiques ont mené à la réalisation d'une analyse de la situation actuelle de l'intervention en matière de surveillance des effets secondaires suivant l'immunisation (ESSI) dans plusieurs provinces.

Objectifs : Mettre en évidence les aspects de l'analyse de situation du système de surveillance des ESSI de Santé Manitoba, Vie saine et Aînés (SMVSA) et démontrer comment des techniques commerciales courantes pourraient être appliquées de manière constructive à un programme provincial de surveillance de l'innocuité des vaccins.

Méthodologie : L'analyse de situation du système de surveillance des ESSI au Manitoba a été élaborée au moyen d'une analyse FFPM (forces, faiblesses, possibilités, menaces) et repose sur les priorités en matière de sécurité des vaccins de la Stratégie nationale d'immunisation. La stratégie a été élaborée en appliquant la matrice FFPM.

Résultats : Treize stratégies ont été élaborées de façon à utiliser les forces pour soit tirer parti des possibilités soit éviter les menaces, à profiter des possibilités pour surmonter les faiblesses, ou à rectifier les faiblesses pour éliminer les menaces. Ces stratégies comprenaient l'élaboration de divers outils et ressources, dont la plupart sont soit activement en cours soit terminés.

Conclusion : L'analyse FFPM et la matrice FFPM ont permis à SMVSA d'accroître la capacité de son programme de sécurité des vaccins.

\section{Introduction}

À la fin avril 2009, l'Organisation mondiale de la Santé (OMS) a annoncé l'apparition d'un nouveau virus grippal A (1). En réponse, de nouveaux vaccins contre la souche de la grippe pandémique devaient être introduits rapidement, et peu de données sur l'innocuité chez les humains étaient disponibles. À ce moment-là, le Canada a activé son Plan de lutte contre la pandémie d'influenza dans le secteur de la santé (2). Bien que ce document traite des questions concernant les vaccins, il décrivait peu la sécurité des vaccins.

À la fin de la pandémie, une nouvelle version du Groupe de travail sur la vaccinovigilance (3) qui comprenait une expertise médicale et épidémiologique accrue - a commandé une évaluation de l'intervention en matière de surveillance des ESSI fédéraux, provinciaux et territoriaux (4). Conformément à ces travaux, en octobre 2010, Santé Manitoba, Vie saine et Aînés ont signé un protocole d'accord avec l'Agence de la santé publique du Canada pour entreprendre le projet pilote sur le modèle de meilleures pratiques liées à la sécurité des vaccins. Le projet pilote sur le modèle de meilleures pratiques visait à combler les écarts entre la pratique actuelle et les priorités en matière de sécurité des vaccins ou les objectifs précis énoncés dans la Stratégie nationale 
d'immunisation (5) pour optimiser le système de sécurité des vaccins, maintenir la confiance du public et des professionnels concernant la sécurité des vaccins, et répondre aux préoccupations croissantes soulevées par le mouvement anti-vaccination.

Deux outils d'affaires communs ont été utilisés pour déterminer la façon de combler ces écarts : l'analyse FFPM (forces, faiblesses, possibilités, menaces), et la matrice FFPM (forces, faiblesses, possibilités, menaces).

L'analyse FFPM est une façon simple de procéder à une vérification des facteurs qui touchent une organisation. Elle facilite l'évaluation des forces et des faiblesses internes d'une organisation - dans ce cas, les FFPM. De même, l'analyse FFPM facilite l'examen des possibilités et des menaces, qui représentent des facteurs de l'environnement externe. Il est impératif de faire une distinction entre les facteurs internes et les facteurs externes, puisque l'organisation a naturellement davantage de contrôle sur les facteurs internes que les facteurs externes. Toute évaluation de la situation actuelle d'une organisation doit prendre en considération les problèmes extrinsèques et les problèmes intrinsèques.

La matrice FFPM est un outil utile pour élaborer des stratégies ou des actions requises liées à la gestion de la santé (6) et va au-delà de l'analyse FFPM (7). Elle permet de faire correspondre systématiquement les différents éléments énumérés dans l'analyse FFPM, montrant les relations parmi les facteurs internes et externes; aidant ainsi les planificateurs à élaborer des mesures qui utilisent des forces pour soit tirer parti des possibilités soit éviter les menaces, qui profitent des possibilités pour surmonter les faiblesses, ou qui rectifient les faiblesses pour éliminer les menaces.

Selon une analyse documentaire de MEDLINE utilisant les termes de recherche " FFPM », " immunisation », et " vaccin », bien que ces techniques aient été utilisées partout dans le monde dans le cadre de la planification stratégique pour les programmes d'immunisation, il n'y a que quelques articles scientifiques décrivant leur application dans ce secteur qui ont effectivement été publiés. Par exemple, un ou deux outils ont été utilisés pour régler les questions liées aux politiques de pénurie de vaccins aux États-Unis (7) et orienter un programme de revaccination antivariolique en Israël (8).

Les objectifs de cette étude visaient à mettre en évidence les aspects de l'analyse de situation du système de surveillance des ESSI de Santé Manitoba, Vie saine et Aînés et à démontrer comment des techniques commerciales courantes pourraient être appliquées de manière constructive à un programme provincial de surveillance de la sécurité des vaccins.

\section{Méthode}

Dans le cadre de l'analyse de situation actuelle du programme de surveillance des ESSI de Santé Manitoba, Vie saine et Aînés au début de ce projet pilote, l'analyse FFPM a été effectuée pour définir les différents facteurs touchant l'organisation. Ces facteurs ont été déterminés selon les réponses aux questionnaires envoyés à divers professionnels de la santé participant à la surveillance des ESSI, y compris les gestionnaires des salles d'urgence des hôpitaux, les gestionnaires des établissements de soins de longue durée, les épidémiologistes, les médecins hygiénistes régionaux, les autres médecins, et les coordonnateurs de la vaccination. Par la suite, la matrice FFPM a été appliquée pour élaborer ou classer par ordre de priorité des stratégies déterminées en consultation avec un groupe consultatif sur la sécurité des vaccins composé du personnel provincial de surveillance des ESSI et des experts en maladies infectieuses. 


\section{Résultats}

Une liste des forces, des faiblesses, des possibilités et des menaces a été établie (voir le tableau 1). L'application de la matrice FFPM à cette liste a permis l'identification des stratégies, qui a nécessité l'élaboration de divers outils et ressources.

Tableau 1 : Liste des forces, faiblesses, possibilités et menaces (FFPM) pour le modèle des meilleures pratiques liées à la sécurité des vaccins de Santé Manitoba, Vie saine et Aînés ${ }^{1}$

\begin{tabular}{|c|c|}
\hline INTERNE & EXTERNE \\
\hline $\begin{array}{cl}\text { Forces } & \\
\text { - } & \text { Formulaire de déclaration existant } \\
\text { - } & \text { Disponibilité des bases de données } \\
\text { - } & \text { Pdministratives } \\
& \text { Procédure existante d'évaluation des risques } \\
\text { - } & \text { Site Web omniprésent du gouvernement } \\
& \text { provincial } \\
\text { - } & \text { Mandat et crédibilité à l'échelle provinciale }\end{array}$ & 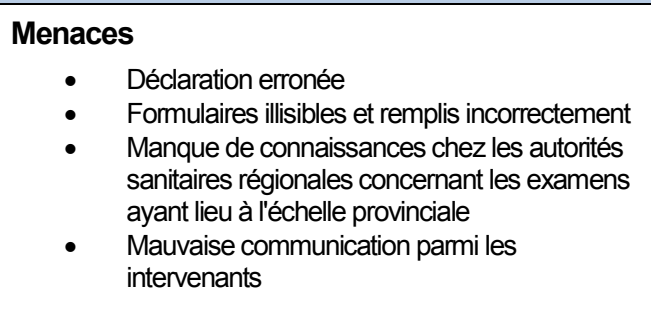 \\
\hline $\begin{array}{l}\text { Faiblesses } \\
\text { - } \quad \text { Aucun objectif de surveillance clairement défini } \\
\text { et documenté } \\
\text { - } \text { Les procédures d'opérations normalisées } \\
\text { (PON) disponibles ne comprennent pas de } \\
\text { descriptions de rôles et responsabilités du } \\
\text { personnel de surveillance clé } \\
\text { - Aucun processus visant à classer ou appliquer } \\
\text { les définitions de cas } \\
\text { - Aucun processus défini pour le suivi et la } \\
\text { déclaration des résultats pour les événements } \\
\text { graves } \\
\text { Absence de commentaires de la province à } \\
\text { l'intention des régions concernant les } \\
\text { déclarations soumises } \\
\text { - Aucun système en place pour effectuer une } \\
\text { analyse solide et importante des données } \\
\text { Aucune orientation concernant la validation des } \\
\text { données } \\
\text { La fonction d'impression de la base de données } \\
\text { n'est pas très utile } \\
\text { - Système fragmenté d'information sur } \\
\text { l'immunisation } \\
\text { Base de données non accessible à l'échelle } \\
\text { régionale } \\
\text { Aucune preuve de lignes directrices sur le } \\
\text { processus d'examen } \\
\text { Aucune source de référence provinciale pour } \\
\text { les médecins hygiénistes }\end{array}$ & 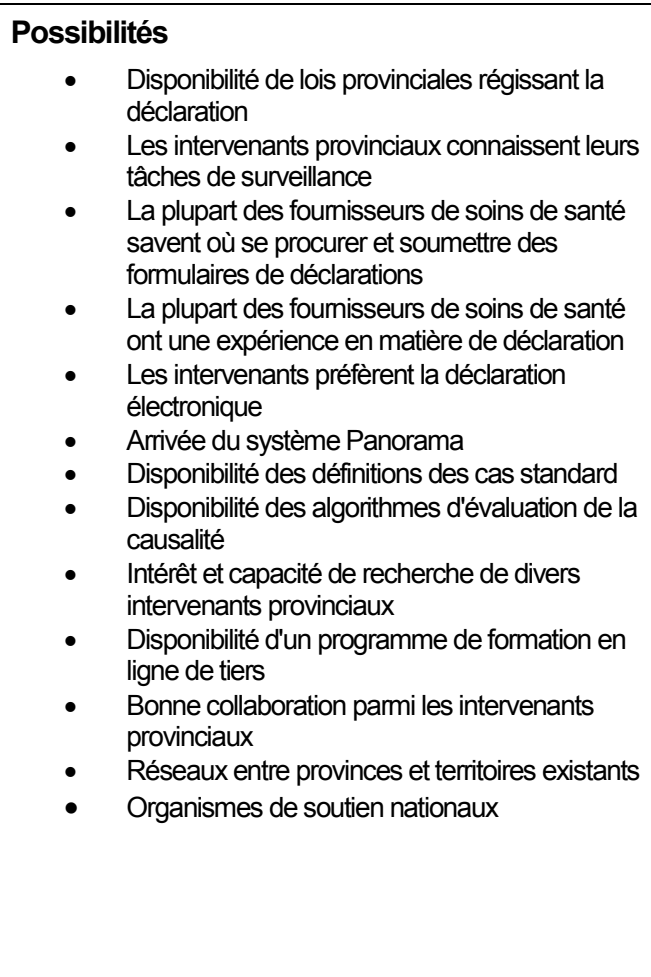 \\
\hline
\end{tabular}

${ }^{1}$ SMVSA - Santé Manitoba, Vie saine et Aînés

\section{Ressources indiquées dans les stratégies des forces et des possibilités}

Programme d'éducation en ligne aux ESSI. En tirant parti de la crédibilité acquise dans le cadre de son mandat à titre d'organisme officiel élaborant des politiques pour le système de santé publique de la province, SMVSA est mesure de demander aux responsables de l'éducation continue des professionnels un accès gratuit au cours existant de formation sur l'immunisation en ligne (9). Ce cours comprend un module sur les ESSI. 


\section{Ressources indiquées dans les stratégies des faiblesses et des possibilités}

Document sur les rôles et les responsabilités en matière de surveillance des ESSI. Ce document repose sur le fait que le Manitoba dispose de lois provinciales obligeant les fournisseurs de soins de santé à déclarer les ESSI. II prend aussi en considération la collaboration et la bonne volonté des intervenants provinciaux.

Outil d'évaluation de Brighton. Cet outil a été élaboré par l'Agence de la santé publique du Canada, et adapté par SMVSA, selon les définitions de cas d'ESSI établies par Brighton Collaboration (10).

Guide provincial d'évaluation de la causalité des ESSI. Ce guide décrit un processus provincial pour le suivi des résultats des événements graves en fournissant une procédure normalisée propre au Manitoba pour établir avec certitude l'existence d'un lien de causalité potentiel de l'administration d'un vaccin avec un ESSI qui répond à un ou à plusieurs critères de gravité. II respecte l'algorithme de l'évaluation clinique de la sécurité vaccinale (11) et est actuellement utilisé de façon active pour évaluer les ESSI individuels. Simultanément, il est continuellement élaboré, s'inspire du manuel de l'utilisateur portant sur l'évaluation de la causalité des ESSI de I'Organisation mondiale de la Santé (12), et sert principalement à développer l'algorithme afin qu'il comprenne la façon de gérer les ESSI à l'échelle de la population.

Méthodologie de détection des signes des ESSI. Cette méthodologie dépendra du résultat de la recherche menée par l'Université du Manitoba au nom de SMVSA en ce qui concerne les niveaux naturels et la validation des signes des ESSI. En outre, Public Health Intelligence for Disease Outbreaks, un outil statistique conçu par le Centre de contrôle des maladies de la Colombie-Britannique, dont SMVSA se sert déjà pour diffuser des alertes en cas d'éclosions de certaines maladies à déclaration obligatoire, pourrait être utilisé.

Outil d'ESSI en ligne directement lié à une base de données. Étant donné la préférence des intervenants pour la déclaration électronique des ESSI, l'utilisation du projet pancanadien de gestion et de surveillance des maladies transmissibles de la santé publique (Panorama) est étudiée pour corriger un système fragmenté d'information sur l'immunisation en ayant une seule base de données pour la vaccination des clients, les stocks de vaccins et les dossiers sur la surveillance des ESSI.

Aide en matière de gestion des ESSI. Cette aide comblerait le manque de sources de référence provinciale pour orienter les médecins hygiénistes en formulant des recommandations de gestion des ESSI et pourrait imiter des exemples provenant des autres provinces et territoires, comme la section des ESSI du manuel d'immunisation du Centre de contrôle des maladies de la Colombie-Britannique (13).

\section{Ressources indiquées dans les stratégies des forces et des menaces}

Formulaire amélioré de déclaration des ESSI. Une mise en page modifiée du formulaire de déclaration jugée plus intuitive pour les professionnels de la santé a été prise en considération pour éviter ou limiter certaines erreurs commises couramment lorsqu'on remplit le formulaire.

Outil d'évaluation des risques des ESSI. Cet outil sera utilisé pour évaluer les risques et l'incidence potentielle d'une alerte ESSI sur le programme d'immunisation. II se fonde sur la procédure existante d'évaluation des risques de SMVSA pour les alertes de santé publique qui fournit une approche structurée en matière de coordination des mesures initiales et de communications après la réception d'un message d'alerte indiquant le risque potentiel pour la santé humaine.

Page des ESSI sur le site Web du gouvernement provincial. Cette page Web (14) peut servir d'outil de diffusion des renseignements et des ressources liés aux ESSI en raison de ce qui est perçu comme une mauvaise communication répandue parmi divers intervenants provinciaux de surveillances des ESSI. 


\section{Ressources indiquées dans les stratégies des faiblesses et des menaces}

Rapports périodiques de surveillance des ESSI. Ces rapports sont considérés comme une façon d'atténuer une faiblesse liée aux rétroactions, ou à leur absence, de la part des provinces aux régions, au sujet de rapports d'ESSI présentés par les régions, car ils éliminent la menace perçue pour les régions sanitaires qui ne sont pas avisées des examens exécutés à l'échelle provinciale.

Fonction d'impression de la base de données des ESSI. Cette fonction permet l'impression d'une base de données individuelles en affichage Formulaire (par opposition à la fonction d'impression en affichage par ligne dans Access, qui n'est pas très pratique), de sorte qu'un nouvel exemplaire du formulaire des ESSI est généré directement à partir de la base de données provinciales et envoyée par télécopieur au système canadien de surveillance des ESSI de l'Agence de la santé publique du Canada (et aux autres, comme les médecins hygiénistes qui en ont besoin), freinant ainsi la dégradation progressive des rapports papiers, ainsi que les impressions et les envois par télécopieur répétés au fur et à mesure que les acteurs prennent part au processus de déclaration (déclarant $\rightarrow$ médecin hygiéniste $\rightarrow$ SMVSA $\rightarrow$ Agence de la santé publique du Canada).

Procédure d'opération normalisée (PON) et guide d'utilisateur liés aux ESSI. Cette procédure et ce guide comblent le besoin de documents d'orientation sur le processus de déclaration : le guide d'utilisateur met principalement l'accent sur les déclarants des ESSI et la procédure d'opération normalisée est surtout axée sur le personnel de surveillance au sein de SMVSA. Ils devraient aider à éviter ou à limiter les déclarations incohérentes.

Les ressources élaborées à partir de la matrice FFPM sont résumées dans le tableau 2.

Tableau 2 : Aperçu de la matrice FFPM $^{1}$ pour le modèle des meilleures pratiques liées à la sécurité des vaccins de SMVSA ${ }^{2}$

\begin{tabular}{|c|c|c|c|}
\hline & & & INTERNE \\
\hline & & Forces & Faiblesses \\
\hline \multirow[t]{2}{*}{ EXTERNE } & Possibilités & $\begin{array}{l}\text { - Programme d'éducation } \\
\text { en ligne aux ESSI }\end{array}$ & 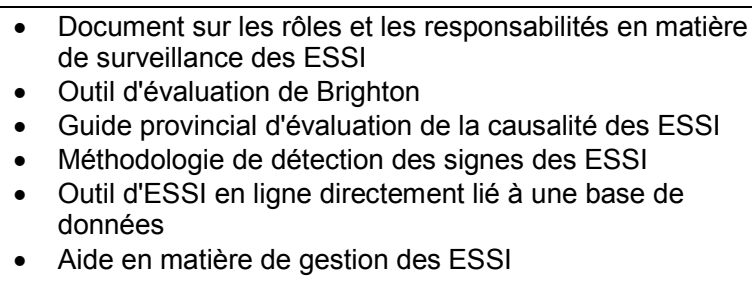 \\
\hline & Menaces & 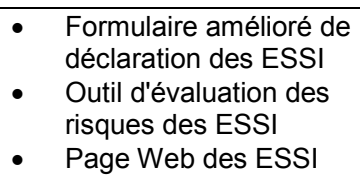 & $\begin{array}{l}\text { - Rapports périodiques de surveillance des ESSI } \\
\text { - Fonction d'impression de la base de données des ESSI } \\
\text { - PON et guide d'utilisateur liés aux ESSI }\end{array}$ \\
\hline
\end{tabular}

FFPM - Forces, faiblesses, possibilités et menaces

${ }^{2}$ SMVSA - Santé Manitoba, Vie saine et Aînés 


\section{Discussion et conclusion}

La matrice FFPM et l'analyse FFPM sont des outils élaborés initialement pour le secteur des affaires. Utilisées ensemble, elles peuvent fournir une approche puissante en matière de planification dans le domaine de la santé publique. SMVSA a profité des connaissances tirées de l'application de ces techniques commerciales qui ont ouvert la voie à l'élaboration des outils et des ressources nécessaires pour mettre en place, on l'espère, un solide programme de sécurité des vaccins qui peut répondre aux défis actuels et aux nouveaux défis lancés par l'expansion continue de son programme d'immunisation global. La plupart de stratégies précédentes sont soit activement en cours, soit terminées et continuellement améliorées. Les stratégies concernant l'utilisation de Panorama, la mise en place d'une aide en matière de gestion et la révision du formulaire de déclaration sont en suspens ou n'ont pas encore commencé.

\section{Remerciements}

Le projet pilote du modèle des meilleures pratiques liées aux ESSI de Santé Manitoba, Vie saine et Aîné a été financée par l'Agence de la santé publique du Canada. Nous tenons à remercier tout spécialement la $D^{\text {re }}$ Barbara Law, chef de la Section de la sécurité des vaccins de l'Agence de la santé publique du Canada, pour son aide et son orientation indéfectibles.

\section{Conflit d'intérêts}

Aucun

\section{Références}

(1) Centre des médias de l'Organisation mondiale de la Santé (OMS). Déclaration du Directeur général de l'OMS, Dr Margaret Chan. Grippe A(H1N1): le niveau d'alerte pandémique passe en phase 6. Genève : OMS; 11 juin 2009 [8 avril 2013]. http://www.who.int/mediacentre/news/statements/2009/h1n1_pandemic_phase6_20090611/fr/

(2) Agence de la santé publique du Canada (ASPC). Plan canadien de lutte contre la pandémie d'influenza dans le secteur de la santé. Ottawa : ASPC; 2006.

(3) Ahmadipour, N., Toth, E., Law, B.J. Groupe de travail sur la vaccinovigilance du Canada. Relevé des maladies transmissibles au Canada, vol. 40(suppl. 3):S40-44, 4 décembre 2014.

One World Inc., editor. Draft meeting proceedings: Vaccine Vigilance Task Group (VVTG); 2010 Mar 30-31. Ottawa: Public Health Agency of Canada [inédit]. http://www.phac-aspc.gc.ca/publicat/nis-sni-03/index-fr.php

(4) One World Inc., editor. Draft meeting proceedings: Vaccine Vigilance Task Group (VVTG); 2010 Mar 30-31. Ottawa: Public Health Agency of Canada [inédit].

(5) Comité consultatif sur la santé de la population et la sécurité de la santé. Stratégie nationale d'immunisation : Rapport final 2003. Rapport du Comité consultatif FPT sur la santé de la population et la sécurité de la santé (CCSPSS) à la Conférence des sous-ministres FPT de la santé. Ottawa : Agence de la santé publique du Canada; 2004. http://www.phac-aspc.gc.ca/publicat/nis-sni-03/index-fra.php

(6) Proctor T. Strategic marketing management for health management: cross impact matrix and TOWS (threats, opportunities, weaknesses, strengths). Journal of Management in Medicine. 2000; 14(1):47-56. Epub 2001/02/24. PubMed PMID: 11183998.

(7) Uscher-Pines L, Barnett DJ, Sapsin JW, Bishai DM, Balicer RD. A systematic analysis of influenza vaccine shortage policies. Public Health. 2008; 122(2):183-91. Epub 2007/09/11. doi: 10.1016/j.puhe.2007.06.005. PubMed PMID: 17825858.

(8) Huerta M, Balicer RD, Leventhal A. SWOT analysis: Strengths, weaknesses, opportunities and threats of the Israeli smallpox revaccination program. The Israel Medical Association Journal. 2003; 5(1):42-6. Epub 2003/02/21. PubMed PMID: 12592958.

(9) Société canadienne de pédiatrie.Programme de formation sur les compétences en matière d'immunisation [consulté en 2014]. 
http://www.advancingpractice.com/p-68-immunization-competencies-education-program.aspx

(10) Brighton Collaboration. Case Definitions [consulté en 2014].

https://brightoncollaboration.org/public/resources/standards/case-definitions.html

(11) Halsey NA, Edwards KM, Dekker CL, Klein NP, Baxter R, LaRussa P, et al. Algorithm to assess causality after individual adverse events following immunizations. Vaccine. 2012; 30:5791-8.

(12) Global Advisory Committee on Vaccine Safety. Causality assessment of an adverse event following immunization (AEFI): User manual for the revised WHO classification. Geneva: World Health Organization; 2013.

(13) British Columbia Centre for Disease Control (BCCDC). Communicable Disease Control Manual. Chapter 2. Immunization. Section IX-Adverse Events Following Immunization. Vancouver, BC: BCCDC; 2014 Jan [consulté le 24 juillet 2014]. http://www.bccdc.ca/NR/rdonlyres/D8098B26-CD7B-4A65-914CAA98B15CC004/0/SectionIX_AdverseEventsFollowingImmunizationsJanuary2014.pdf

(14) Manitoba Health, Healthy Living and Seniors. Communicable Disease Control. Adverse Events Following Immunization (AEFI). Winnipeg, MB: Province of Manitoba [consulté en 2014]. http://www.gov.mb.ca/health/publichealth/cdc/div/aefi.html 